\title{
Review of mastectomy in the department of gynecology at the Treichville teaching hospital, Abidjan-Cote d'Ivoire
}

\author{
Yao Ignace N'guessan ${ }^{1 *}$, Dia Jean-Marc Lamine', Toure Moctar ${ }^{2}$, Saki Téa Corneille ${ }^{1}$, Tela \\ Simo Gilbert ${ }^{1}$, Oyelade Mouhideen Adunfé ${ }^{1}$, Okon Gérard ${ }^{1}$, Guie Yéret Privat ${ }^{1}$, \\ Anongba Danho Simplice ${ }^{1}$
}

\begin{abstract}
${ }^{1}$ Department of Obstetrics and Gynecology, ${ }^{2}$ Department of Cancerology, Treichville Teaching hospital, Abidjan-Côte
\end{abstract} d'Ivoire

Received: 13 February 2018

Accepted: 09 March 2018

\section{*Correspondence:}

Dr. Yao Ignace N'guessan,

E-mail: yaoignacen@yahoo.fr

Copyright: () the author(s), publisher and licensee Medip Academy. This is an open-access article distributed under the terms of the Creative Commons Attribution Non-Commercial License, which permits unrestricted non-commercial use, distribution, and reproduction in any medium, provided the original work is properly cited.

\section{ABSTRACT}

Background: Mastectomy plays a key role in the management of breast cancer in our regions of sub-Saharan Africa. Because the advanced forms represent the essential stages of the diagnosis and some therapeutic means remain unavailable. Objective of this study was to report the experience of the practice of mastectomy in the treatment of breast cancer in our service.

Methods: This is a retrospective and prospective, descriptive study conducted from January 1, 2013 to May 31, 2017 (age 05) at the University Hospital of Treichville involving 56 breast cancer patients who had undergone a mastectomy.

Results: The frequency of the mastectomy was $28.5 \%$ and the average age of our patients was 48 years old. The majority of patients had an average socio-economic level (66\%). 85.6\% of our cancers discovered at advanced stages (T3 and T4). Adenocarcinoma accounted for $96 \%$ and infiltrating ductal carcinoma $82 \%$ of adenocarcinoma.

Patey mastectomy associated with axillary dissection was performed in $96.4 \%$ and simple mastectomy in $3.5 \%$. The results of axillary dissection reported lymph node invasion in 38 patients; With an average number of lymph nodes taken from 6.1 and an average number of ganglia affected is 3. Neoadjuvant chemotherapy was administered in $96.4 \%$ and adjuvant chemotherapy in $91 \%$. Radiotherapy was performed in $34 \%$. Complications were dominated by lymphoceles in $34 \%$ of cases. The 5-year survival of patients operating in the service is $37.8 \%$.

Conclusions: Mastectomy is at the forefront of breast cancer surgery in our service. She is supervised by chemotherapy. Radiotherapy remains inaccessible for most patients. Early detection would lead to conservative treatment and a reduction in the postoperative complication rate.

Keywords: Breast cancer, Complications, Mastectomy

\section{INTRODUCTION}

Breast cancer is a common condition that today represents a real public health problem around the world. ${ }^{1}$ In developed countries, routine screening, early diagnosis and therapeutic progress have improved prognosis. ${ }^{2,3}$ In
Europe, breast cancer mortality is declining, with a decrease of between $8 \%$ and $10 \%$ between 1987 and 2002. ${ }^{4}$

In the developing countries, however, mortality remains high. In Ivory Coast, the incidence of breast cancer has 
been 33.7 per 100,000 women since 2012 and breast cancer mortality is estimated at $54 \% .^{5,6}$ In the countries, surgery remains the main therapeutic weapon and is dominated by mastectomy associated with axillary dissection. This is because of the advanced forms that represent the essential stages of the diagnosis. Author propose in this study to take stock of 5 years of management of breast cancer in our service.

\section{METHODS}

This work is a transversal, descriptive study conducted between January 2013 to May 2017 in the obstetrics and gynecology department of the University Hospital of Treichville. The study focused on patients treated for breast cancer in the senology unit during the period. Author included all patients with breast cancer who had a mastectomy. Patients who had incomplete records and those whose mastectomy was performed out of service were not included. The data were collected using a standardized survey form from the Patient Survey, the Patient Medical Observation Record, the Consultation Records, the Chemotherapy Registries, operational record books and patient follow-up log. The parameters were collected with the Epi Data software and analyzed with stata software. Data entry (text and tables) was done using Word and Excel software.

\section{RESULTS}

\section{Frequency}

During the study period, 1168 patients consulted for breast disease. He was diagnosed with breast cancer in 221 patients, or $19 \% .63$ had a mastectomy in our service, an average frequency of $28.5 \%$ with extremes of $17.6 \%$ in 2014 and $40.4 \%$ in 2016 (Table 1).

\begin{tabular}{|ll|}
\hline Year & Frequivency $(\%)$ \\
\hline 2013 & 26.7 \\
\hline 2014 & 17.6 \\
\hline 2015 & 24.4 \\
\hline 2016 & 40.4 \\
\hline 2017 (January-May) & 40 \\
\hline
\end{tabular}

Table 1: Frequency evolution during the study period.

\section{Socio-demographic characteristics}

The socio-demographic characteristics are specified in Table 1.34 patients, $61 \%$ were under 50 in the series and $66.1 \%$ had an average socioeconomic level.

\section{Clinical and histological characteristics}

There was a slight predominance of cancer in the right breast with $51.7 \%$ and a predominance of $\mathrm{T} 3$ and $\mathrm{T} 4$ stages in $85.6 \%$ of cases. $52 \%$ of the patients were N0 and $98.2 \%$ had no metastases at the time of diagnosis.
Table 2: Socio-demographic characteristics.

\begin{tabular}{|lll|}
\hline Characteristic & Total & Percentage \\
\hline Age (year) & & \\
\hline $30-39$ & 9 & 16 \\
\hline $40-49$ & 25 & 45 \\
\hline $50-59$ & 17 & 30 \\
\hline $60-69$ & 5 & 9 \\
\hline Socio-economic level & & \\
\hline High & 8 & 14,3 \\
\hline Average & 37 & 66,1 \\
\hline Low & 11 & 19,6 \\
\hline Parity & & \\
\hline Nulliparous & 6 & 10,7 \\
\hline Primipare & 5 & 8,9 \\
\hline Few previous deliveries & 24 & 42,8 \\
\hline Multiparous & 21 & 37,5 \\
\hline Total & 56 & 100 \\
\hline
\end{tabular}

Adenocarcinomas were the most common histological type with $96 \%$ of cases. The histological features are described in Table 3. The histoprognosis grade was dominated by grade II with $71.7 \%$. These results were obtained after the histological examination of the microbiopsy and confirmed by pathological anatomy examination of the mastectomy and axillary dissection.

Table 3: Distribution of patients by histological type.

\begin{tabular}{|lll|}
\hline Histological type & No. & Percentage \\
\hline Adenocarcinomas & 54 & 96 \\
\hline Invasive ductal carcinoma & 44 & 81.5 \\
\hline Lobular carcinoma & 5 & 9.3 \\
\hline Mixed infiltrative carcinoma & 3 & 5.6 \\
\hline Mucinous carcinoma & 1 & 1.9 \\
\hline Micropapillary carcinoma & 1 & 1.9 \\
\hline Sarcomas & 2 & 4 \\
\hline Total & 56 & 100 \\
\hline
\end{tabular}

\section{Treatment}

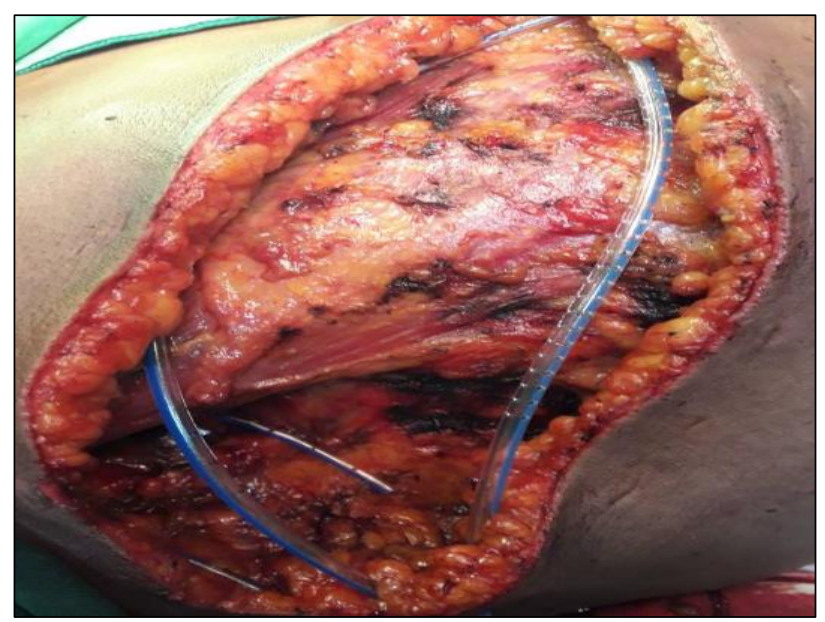

Figure 1: Patey mastectomy with axillary dissection. 
Patey mastectomy associated with axillary dissection was the basic treatment of our cancers in $96.4 \%$ of cases (Figure 1). Two patients were not able to benefit from axillary dissection because the surgery was a mastectomy of cleanliness. The average number of lymph nodes removed during the dissection was $6.18 \pm 4.64$ ganglia with extremes ranging from 2 to 21 lymph nodes. Ganglionic invasion was noted 38 patients $(70.3 \%)$

Intraoperative complications are shown in Table 4. The most common postoperative complication was axillary lymphocele in $34 \%$ of cases and arm lymphoedème in $10.7 \%$ of cases.

Table 4: Distribution of patients according to the complications.

\begin{tabular}{|lll|}
\hline Complications & Number & Percentage \\
\hline Per-operative hemorrhage & 14 & 25.0 \\
\hline Immediate post-operative & & \\
\hline Hematoma & 1 & 1.8 \\
\hline Sensory disturbances & 3 & 5.4 \\
\hline Secondary post-operative & & \\
\hline Axillary lymphocele & 19 & 34 \\
\hline Lymphœdema & 6 & 10.7 \\
\hline Abscess under the parietal & 1 & 3.4 \\
\hline Sequential pain & 3 & 5.4 \\
\hline
\end{tabular}

Fifty-four patients (96.4\%) received neoadjuvant chemotherapy and 51 patients $(91 \%)$ received adjuvant chemotherapy. The protocol used was 3 or 4 courses of FEC100 in neoadjuvante and 3 or 4 courses of Taxane monotherapy in adjuvant. Only 19 patients (34\%) were able to radiotherapy, and all were out of our country because of the absence of radiotherapy. The 5-years survival of patients operating in the service is $37.8 \%$.

\section{DISCUSSION}

\section{Frequency}

In this study the frequency of mastectomy accounted for $28.5 \%$ of diagnosed breast cancers that should have undergone surgical treatment. This very important difference can be explained by several reasons, namely that many patients were lost to follow-up after the diagnosis, because of the cost of treatment on the one hand, which was not available to certain patients, and others because of the stage of the diagnosis which for the most part was late.

Some patients have also been treated after their diagnosis in the cancer ward. These patients were not included in this study.

\section{Socio-demographic characteristics}

The most affected age group was between 40 and 49 years old and accounted for $45 \%$ of cases. The average age was 48.1 years with extremes ranging from 32 to 69 years old. Our results are close to those of EFFI (45.21 years) at the national level. ${ }^{7}$

Elsewhere in Africa B. Traoré in Guinea reported an average age very close to ours with 46.2 years. ${ }^{8} \mathrm{H}$ Ben Gobrane in Tunisia reports an average age slightly higher than ours with 49.7 years. ${ }^{9}$

Middle-class patients accounted for $66.1 \%$ of present study. This trend seems to be the same in most of present developing countries. The low purchasing power of patients makes it difficult to fully care for patients in our countries.

\section{Clinical characteristics}

Inflammatory cancers accounted for $19.6 \%$ of the cases in our study. N'Koua-Bon in Brazzaville (Congo) found $15.16 \%$ in his series. ${ }^{10}$

The literature in developed countries reports that inflammatory breast cancer represents a fairly rare form of breast cancer with an incidence of between $1 \%$ and $6 \% .^{11}$

This high frequency in the series is explained by the absence of screening and the delay of diagnosis. For the same reasons, the tumors are found at stage $\mathrm{T} 3$ and $\mathrm{T} 4$ $(85.7 \%)$ and stage $\mathrm{N} 1(46 \%)$. Our results are close to those of ZONGO, in Burkina-Faso, which found $82.7 \%$ of patients who were at stage T3 and T4. S Mayi-Tsonga et al in Gabon found $70 \%$ at these same stages. ${ }^{12,13}$

Adenocarcinoma accounted for $96 \%$ and sarcomas $4 \%$ in our study. These results are superimposable to those found in the literature of grade II tumors accounted for $71.7 \%$ in the series. ${ }^{14,15}$ Ahmed SB found a lower grade II rate in his series $(52.1 \%){ }^{16}$

This grade corresponds to an intermediate position and is considered Grade III in decision-making. The higher the grade, the more aggressive the tumor is and the poorer prognosis.

\section{Treatments}

\section{Type of intervention}

In this study, $96.4 \%$ of patients had undergone a Patey mastectomy with axillary dissection. Radical treatment is the most used in African series more than $60 \%$ of cases because of the late diagnosis and lack of radiotherapy. ${ }^{17}$

In developed countries, however, mastectomy accounts for only $30.5 \%$ of breast cancer procedures. ${ }^{18}$ In France in particular, while surgery remains the first therapeutic act in breast cancer, 60 to $70 \%$ of patients benefit from conservative surgery. ${ }^{19}$ 


\section{Associated treatments}

Chemotherapy has been associated with mastectomy in more than $90 \%$ of cases. This association has been the mainstay of patient management in the context. ${ }^{20}$

Chemotherapy significantly improves the survival of patients in advanced stages. $^{21}$

Radiotherapy was performed only in $40 \%$ of cases. This rate is still higher than that of Agah in Côte d'Ivoire $(16.7 \%)$ and Zongo in Burkina-Faso (8.2\%). ${ }^{12,22}$

These results demonstrate the difficulty for the majority of patients to perform radiation therapy. Radiotherapy does not exist in most of our sub-Saharan African countries. Patients are sometimes obliged to go to North Africa or Europe to perform radiotherapy. Which is not within the reach of all the patients.

\section{Surgical suites}

Perioperatively, hemorrhagic complications (25\%) were marked by bleeding difficult to control at the time of axillary dissection due to fixed lymphadenopathy. Complications occurring within 72 hours after the procedure were represented in $75 \%$ of cases by sensory disorders. The results are superimposable to the data of the literature. ${ }^{23}$ The axillary lymphocele (34\%) and lymphedema $(10.7 \%)$ found in our series are comparable to the postoperative complications described in the literature. $^{24}$

Early detection of breast cancer and sentinel lymph node involvement would significantly improve the morbidity associated with deep and systematic axillary dissection in all operated patients. The 5-year survival of patients operating in the service is $37.8 \%$.

This rate is much lower than the developed country, but it remains higher than those of Gueye in Senegal which is $31.8 \%$. This difference is explained by the study population of Gueye, which deals with inflammatory cancers that are bad prognosis. 25

\section{CONCLUSION}

Mastectomy is at the forefront of breast cancer surgery in our service. This rate remains relatively low because of many patients lost to follow-up. Tumours are discovered at late stages with a high rate of inflammatory form. This surgery is supervised by neoadjuvant and adjuvant chemotherapy. Radiotherapy remains inaccessible for most of our patients. Operative complications are dominated by lymphoceles due to axillary dissection. Early detection would lead to conservative treatment and a reduction in the postoperative complication rate. In addition, breast reconstruction should be considered to improve the daily experience of operated patients.
Funding: No funding sources

Conflict of interest: None declared

Ethical approval: The study was approved by the Institutional Ethics Committee

\section{REFERENCES}

1. Latt M. The problem of mammographic screening for breast cancer. Thesis Medicine Abidjan 2001: $\mathrm{N}^{\circ}$ 3003.

2. Coleman MP, Gatta G, Verdecchia A, Esteve J, Sant M, Storm H, et al. EUROCARE-3 summary: cancer survival in Europe at the end of the 20th century. Ann Oncol. 2003;14:v128-49.

3. Kalager M, Zelen M, Langmark F, Adami HO. Effect of screening mammography on breast-cancer mortality in Norway. N Engl J Med. 2010;363:120310 .

4. Levi F, Lucchini F, Negri E, La Vecchia C. Continuing declines in cancer mortality in the European Union. Ann Oncol. 2007;18:593-5.

5. Echimane AK, Ahnoux AA, Adoubi I, Hien S, M'Bra K, D'Horpock A, Diomande M, Anongba D, Mensah-Adoh I, Parkin DM. Cancer incidence in Abidjan, Ivory Coast. Cancer. 2000 Aug 1;89(3):653-63.

6. Ferlay J, Soerjomataram I, Ervik M, Dikshit R, Eser S, Mathers C, et al. Globocan 2012, Cancer Incidence and Mortality Worldwide International Agency for Research on. Cancer. 2013.

7. N'Dah KJ, Doukoure B, Troh E, Koffi KE, Kouamé $\mathrm{AD}$, Effi AB, et al. Epidemiological and Histological Aspects of Women Genital Cancers in Côte d'Ivoire. Open J Obstet Gynecol. 2014 Jun 23;4(09):516.

8. Traoré B, Eric-Douanla D, Diallo Y. The problem of the management of breast cancers from "postoperative diagnosis" to the oncological surgery unit, Donka Hospital, Conakry (Guinea). J Afr Cancer. 2010;2:140-5.

9. Abdallah MB, Achour N, Hsairi M. Prognosis of breast cancer at Salah Azaiez Institute of Carcinology of Tunis. East Mediterr Health J. 2007;13(2):309.

10. Dawood S. International expert cancer: panel on inflammatory breast consensus statement for standardized diagnosis and treatment. Ann Oncol Off J Eur Soc Med Oncol. 2011;22:515-23.

11. Woodward WA, Cristofanilli M. Inflammatory Breast Cancer. Semin Radiat Oncol. 2009;19:256-65.

12. Zongo N. Place of surgery in the management of breast cancer in women at the University Hospital Center Yalgado Ouedraogo. Pan Afr Med J. 2015.

13. Mayi-Tsonga S, Belembaogo E, Meye JF, Ngou JP. Breast cancer in Gabon: epidemiological, diagnostic and therapeutic aspects. J Afr Cancer. 2009;1:11-5.

14. Engbang JP, Essome H, Koh VM, Simo G, Essam JD, Mouelle AS, et al. Breast cancer in Cameroon, histo-epidemiological profile: about 3044 cases. Pan Afr Med J. 2015;21:242. 
15. Bakkali H, Marchal C, lesur-Schwander A, Verhaeghe JL. Breast cancer in women 30 and under. Cancer/Radiotherap. 2003;7:153-9.

16. Ben SA, Aloulou S, Bibi M, Landolsi A, Nouira M, Ben LF, et al. Breast cancer prognosis in Tunisian women: analysis of a hospital series of 729 patients. Sante publique (Vandoeuvre-les-Nancy, France). 2002 Sep;14(3):231-41.

17. Khanfir A, Frikha M, Kallel F, Meziou M, Trabelsi $\mathrm{K}$, Boudawara $\mathrm{T}$, et al. Breast cancer in young women in the south of Tunisia. Cancer radiotherapy. J French Soc Radiotherap Oncol. 2006;10(8):565-71.

18. Anongba DS, Toure S, Tegnan JA, Guié $\mathrm{P}$ et al. Surgical treatment of breast cancer in the department of gynecology and obstetrics chu Treichville. Int $\mathbf{J}$ Gynecol Obstet of Cote d'Ivoire. 2004;27-9.

19. Giard S. Breast cancer and outpatient surgery. edissertations of the National Academy of Surg. 2014;13(3):083-5.

20. Sarre B, Ogougbemy M, Dotou C. Epidemiological, therapeutic and prognostic aspects of breast cancer: about 473 cases collected in Hopital Principal de Dakar. Dakar Med. 2006;51(2):92-6.

21. Yang MT, Rong TH, Huang ZF. Clinical analysis of resecable breast cancer: a report of 6236 cases. Ai Zheng. 2005;24(3):327-31.
22. Agah J. Breast cancer surgery in the Ivorian context: case of the obstetrics and gynecology department of the University Hospital of Treichville. PhD thesis in Medicine, Abidjan, 2014-2015.

23. Kuehn T, Klauss W, Darson M. Long-term morbidity following axillary dissection in breast cancer patients clinical assessment significance for life quality and the impact of demographic, oncologic and therapeutics factors. Breast Cancer Res Treat. 2000;64:275-86.

24. Pogson CJ, Adwani A, Ebbs SR. Seroma following breast cancer surgery. Eur J Surg Oncol. 2003;23:711-7.

25. Gueye M, Kane-Gueye SM, Ndiaye-Gueye MD, Gassama O, Diallo M, Moreau JC. Inflammatory breast cancer: features and outcomes in a Breast Unit in Dakar, Senegal. Int J Reprod Contracept Obstet Gynecol. 2016;5:361-6.

Cite this article as: N'guessan YI, Dia Lamine J, Moctar T, Corneille ST, Gilbert TS, Adunfé OM, et al. Review of mastectomy in the department of gynaecology at the Treichville teaching hospital, Abidjan- Cote d'Ivoire. Int J Reprod Contracept Obstet Gynecol 2018;7:1313-7. 